\title{
Research on Portfolio Risk Prediction Based on Copula-GJR-Skewt Model
}

\author{
Xiangqing wei ${ }^{1, \text { a }}$ \\ ${ }^{1}$ Office of Research on Teaching, Bureau of Education of Guang rao County, Shandong, China \\ alubianinfo@foxmail.com
}

Keywords: Copula, GJR-Skewt; Portfolio Risk; Prediction

\begin{abstract}
For risk prediction of diversified investment portfolio, we use the thick tail and the biased characteristics of GJR-Skewt model to depict a single asset and using Copula model to depict a diversified investment portfolio non-linear correlation structure, simulating the random distribution of financial assets with Monte Carlo method and combining with rolling time window method to conduct the sample dynamic forecast for the future portfolio risk. The empirical results show that Copula-GJR-Skewt model can achieve satisfactory results of predicting the risk of asset returns. For the VaR forecast performance, we use the GJR-Skewt model as the edge distribution functions and even if there is a system error, it can also achieve optimal prediction.
\end{abstract}

\section{Introduction}

Risk management is an important part of the financial regulatory agencies concerned, as the financial derivatives market share continues to increase at the present stage of high risk, the risk of dependence between different markets significantly enhanced which is a financial asset, especially portfolio a higher risk of regulatory requirements. Early scholars multivariate Garch model to describe the relationship between multiple variables, but it is the presence of parameter estimation, multivariate distributions assume limitations on such issues in recent years, Copula function since being first used in risk management and other areas. Due to good mathematical properties, it gets a lot of applications. As a new measurement tool between multiple variables related structures, Copula function describes the rank correlation between random variables, reflecting the relevance under strict monotonic transformation, than the linear the correlation coefficient of the broad scope of application. In addition, Copula distribution function of a single variable that is marginal distribution and joint distribution of multiple variables banded together Copula function application when the joint distribution structure, no pre-edge distribution follows a particular distribution, it can be flexible distribution constructed according to the specific circumstances of different edge. In addition, the edge of Copula distribution parameters and associated structural parameters can be estimated in two stages, with a relatively simple model to estimate the benefits, which makes the application of the model to be more potential [1].

For this reason, Copula model financial risk has been widely applied. Existing studies have shown Copula function with certain volatility model can be effectively applied to a combination of risk management. However, the existing research is still less than certain, based on the above understanding paper proposes to build Copula-GJR-Skewt model to capture the higher moments and correlation characteristics of the portfolio return series on the future risk of the portfolio. GJR-Skewt dynamic prediction model can effectively portray the samples outside the financial time series biased thick tail, volatility clustering and Copula function can be measured by the nonlinear relationship between assets based on Copula-GJR-Skewt model, this paper randomly distributed simulation Monte Carlo simulation method to calculate the financial assets VAR. MC method can measure both the Delta-normal method cannot measure the nonlinear price risk, but also can improve the historical simulation method relies entirely insufficient historical data the same time, the count of the correlation structure of assets and asset volatility conditions between the timing of characteristics, this paper scroll forward one day time window predicted VAR method to predict future trends, in order to improve the prediction accuracy of VAR. In addition, as a comparative 
study, the paper also calculated with several other group volatility models as the marginal distribution of VAR prediction. The empirical results show that, Copula-GJR-Skewt model can be used to predict the risk of asset returns and can achieve satisfactory results compared to several other volatility models. GJR-Skewt model as the edge of the distribution function has the best prediction performance, even in the presence of systematic bias lower.

\section{The Construction and Estimation of Mathematical Model of Financial Assets Portfolio Return Series}

The Construction of AR-GJR-Skewt Model of Asset Portfolio Edge Allocation.Time series of financial assets yield there is often self-correlation, volatility clustering and leverage other features. The so-called leverage effect refers to the residual term yields negative sensitivity to the impact on the market is greater than the positive impact on the market. Engle et-response curves were compared using the information leverage various Garch models that GJR models better characterize the leverage capital gains rate. In view of this, the paper selection of AR (1)-GJR $(1,1)$ model depicts a single volatility return on assets, the mathematical expression model are as follows [2]:

$$
\begin{aligned}
& r_{i, t}=c_{1} r_{i, t-1}+e_{i, t} \\
& e_{i, t}=h_{i, t} \varepsilon_{i, t} \\
& h_{i, t}=\omega_{i, t}+\alpha e_{i, t-1}^{2}+\beta h_{i, t-1}+\lambda D_{t-1} e_{i, t-1}^{2}
\end{aligned}
$$

In which $r_{i, t}$ is at the time of $t$ the return on assets $i, e_{i, t}$ interest rates for new moment $t$ for assumed to follow a particular distributed random variables $\varepsilon_{i, t}, h_{i, t}$ conditional variance. Equation (3), if the coefficient $\beta>0$, then the $t$ day fluctuations in yield has a positive relationship with the former, it is clear the presence of aggregates yields fluctuate day . $D_{t-1}$ to measure the residual effect of leverage indicator function:

$$
D_{t-1}= \begin{cases}1 & \text { if } \varepsilon_{t-1}<0 \\ 0 & \text { if } \varepsilon_{t-1} \geq 0\end{cases}
$$

The Portfolio Assets Joint Distribution Modeling Based on Copula. For the portfolio, the assessment of risk depends not only on fluctuations in univariate or loss, also depends on the correlation of each asset in the portfolio assets. Because it was not consistent with a linear correlation between the traditional multi-dimensional variables most assets the correlation coefficient between some limitations, but Copula function has excellent mathematical properties in this area. according to Theorem Nelson proposed Copula function of the random variables do strictly monotone increasing transformation, the measure of the correlation function derived values are not will change. Therefore, the linear correlation coefficient compared to the rank correlation coefficient derived from Copula function has a broader application [3].

By Sklar theorem, for $\mathrm{N}$-dimensional random variable $x_{1}, x_{2}, x_{3} \cdots, x_{N}$, distribution function if its edges, respectively $F_{\left(x_{1}\right)}, F_{\left(x_{2}\right)}, \cdots F_{\left(x_{N}\right)}$, the joint distribution function is $F\left(x_{1}, x_{2}, x_{3} \cdots, x_{N}\right)$, then there exists a Copula function $\mathrm{C}$, meet

$$
F\left(x_{1}, x_{2}, x_{3} \cdots, x_{N}\right)=\mathrm{C}\left(F_{\left(x_{1}\right)}, F_{\left(x_{2}\right)}, \cdots F_{\left(x_{N}\right)}\right)
$$

In the marginal distribution function $F_{\left(x_{1}\right)}, F_{\left(x_{2}\right)} \cdots F_{\left(x_{N}\right)}$ under continuous conditions, Copula functions can be uniquely identified Essentially, Copula function is subject to random variables $(0,1)$ uniformly distributed as independent variables, marginal distribution structure $(0,1)$ uniform distribution multivariate joint distribution, so Copula function does not limit the selection edge distribution for yields of financial assets, it can be modeled on the edge of the distribution based on the specific characteristics of earnings. In order to obtain the trailing edge of the distribution of individual financial assets in the portfolio, select corresponding Copula function, we can obtain the joint distribution portfolio, and thus the export portfolio of the correlation matrix. This article will use the relationship described having a thick tail structure to facilitate the combination of T-Copula 
function of assets, if $\mathrm{C}$ represents $\mathrm{T}$ - distribution Copula function, indicating that the correlation coefficient matrix, $\mathrm{T}_{\rho, v}$ multi-degree $\rho$ of freedom $v$ for the Student-T function, $T_{v}^{-1} 0$ expressed a degree of freedom $v$ for the inverse function Student-T functions, mathematical expressions dimensional T-Copula function of the $\mathrm{N}$ as follows:

$$
C\left(u_{1}, u_{2}, \cdots u_{N}, \rho, v\right)=\mathrm{T}_{\rho v v}\left[T_{v}^{-1}\left(u_{1}\right), \ldots, T_{v}^{-1}\left(u_{N}\right)\right]
$$

\section{The Empirical Results and Analysis}

The Descriptive Statistics of Assets Return Time Series. This paper selects Chinese CSI 300 Index (HS300), the US S\&P index (SP500), Japan's Nikkei 225 (NKY), the British FTSE 100 index (FTSE) closing index build an international portfolio, investigated the combination yields the risk of data for selected period April 1, 2005 to April 1, 2013, taking into account different national stock trading day of sync or holidays and other reasons, excluding trading day non-overlapping data, obtained after processing 7064 data That 1766 group synchronization data. Data sources for the great wisdom of the database. To compare the models to predict the effect, 1766 sets of data are divided into groups within the 1200 samples and 566-sample data set, the sample data as foreign portfolio VAR prediction test samples.

First, the closing index of the number of processing, set the $t$ day closing price $\mathrm{p}_{t}$, the daily yield $=100 * \ln \left(p_{t} / p_{t-1}\right)$ The descriptive statistics of yield processed data is shown in Table 1.

Table 1 The descriptive statistics of yields

\begin{tabular}{lcccccr}
\hline & M & SD & Kurtosis & Skewness & J-B & \multicolumn{1}{c}{ LM(2) } \\
\hline FTSE & 0.0491 & 1.1838 & -0.1566 & 4.9724 & $145.4203^{* * *}$ & $0.1529[0.000]$ \\
HS300 & -0.0043 & 1.6439 & -0.1701 & 4.9825 & $147.5074 * *$ & $0.1527[0.000]$ \\
NKY & 0.0369 & 1.4056 & -0.5434 & 8.1207 & $999.0427^{* * *}$ & $-0.0695[0.0623]$ \\
SP500 & 0.0734 & 1.2574 & -0.2504 & 6.4429 & $441.2983^{* *}$ & $0.3119[0.0000]$ \\
\hline
\end{tabular}

Note: $1 . * * *$ indicates significant at $1 \%$ level is significant.

2. The square brackets in the ARCH Tests LM statistic P value, LM (2) indicates lag is 2.

From Table 1, the four sets of data are shown in left side morphology (the skewness coefficient is less than 0 ) and kurtosis are larger than normal kurtosis 3, indicating the presence of significant data "fat tail" feature; JB statistic show four sets of data are rejected at $1 \%$ of the normal level of significance; visible, there is a significant "spike fat tail" feature comprehensive data .Arch effect on LM test results prove NKY at $10 \%$ significance level refusal does not exist ARCH the null hypothesis effect, the other three sets of data were $1 \%$ significance level reject the null hypothesis ARCH effect does not exist, so the choice of Garch class model is reasonable [4].

The Forecasting Results of Dynamic VAR-sample. In calculating the portfolio VAR, paper, we consider four assets and other heavy right circumstances, according to the portfolio VAR Monte Carlo simulation method to predict the future 566 days of VAR to rolling time window method. First calculate the portfolio to meet the diverse T-Copula function the rank correlation coefficient matrix. the estimated sample is fixed at 1200 days, followed with the first 1200 days before $t$ based on historical data to calculate the Copula parameters and then calculate the correlation coefficient matrix, where $t=(1201, \ldots, 1766)$ the correlation coefficient, the group received a total of 566 time-varying matrices. Dues to limited space, select the first group and 566 group's rank correlation coefficient matrix shown in Table 2. 
Table 2 The T-Copula rank correlation coefficient matrix

\begin{tabular}{ccccc}
\hline $\begin{array}{l}\text { Group1 } \\
\text { Index }\end{array}$ & FTSE & HS300 & NKY & SP500 \\
\hline FTSE & 1 & 0.1125 & 0.2286 & 0.4505 \\
HS300 & 0.1125 & 1.0000 & 0.1715 & 0.0667 \\
NKY & 0.2286 & 0.1715 & 1.0000 & 0.1235 \\
SP500 & 0.4505 & 0.0667 & 0.1235 & 1.0000 \\
\hline Group566 & & & & \\
Index & FTSE & HS300 & NKY & SP500 \\
\hline FTS & 1.0000 & 0.0968 & 0.2389 & 0.4164 \\
HS300 & 0.0968 & 1.0000 & 0.1584 & 0.0653 \\
NKY & 0.2389 & 0.1584 & 1.0000 & 0.1090 \\
SP500 & 0.4164 & 0.0653 & 0.1090 & 1.0000 \\
\hline
\end{tabular}

When calculated according to varying rank correlation coefficient matrix, using a Monte Carlo simulation randomly distributed into four groups of data. On the first day forecast scenarios were simulated 40,000 times (each data 10,000 times) to give the first day 10,000 times may be distributed, and then calculated separately in accordance with section 1-3 of the calculation steps to obtain a given level of confidence Dir day VAR predictive value, empathy was the next day, the third day ...... total of 566 days of the VAR forecast. That is, for each given confidence level VAR value, for a total of $40000 \times 566=22640000$ times scenario simulation calculations [5].

\section{Conclusions}

This paper uses the Copula-GJR-Skewt model to depict the correlation between the typical facts of single-asset and portfolio and uses rolling time window method to predict the dynamic VAR of portfolio. Positive results can be seen that: for the most representative stock index of Chinese, United States, Japan and Britain, it yields sequences with significant volatility clustering, fat tail and biased characteristics and the GJR-Skewt model can fit these features of the stock index. It should also be seen that the correlation between the portfolios is really complex, distributed to more diverse between related assets to be further validated and it is the next problem to be solved.

\section{References}

[1] Dengshi Huang: Journal of Systems Engineering, Vol. 1 (2008) No 23, p.45-51

[2] Yanhua Wei: Application of Statistics and Management, Vol. 3 (2007) No 26, p.432-439

[3] Zhidong Liu: Quantitative \& Technical Economics, Vol. 9 (2010) No 33, p.147-160

[4] Zhenxiang Wu: System Engineering Theory and Practice, Vol. 3 (2006) No26, p.45-52

[5] Xiaohua Zhou: Journal of Management, Vol. 12 (2012) No15, p.70-78 